\title{
Comunicación
}

\section{Diagnóstico y tratamiento de persistencia de cuarto arco aórtico derecho en un perro}

\author{
Diagnostic and treatment of a persistent fourth right aortic arch in a dog
}

Ysaac Chipayo G. ${ }^{1}$, Juan Olazábal L. ${ }^{2}$, Carlos Huerta M. ${ }^{1}$, Diego Díaz C. ${ }^{1}$

\section{Resumen}

\begin{abstract}
El cuarto arco aórtico persistente es una anomalía congénita que se considera de difícil tratamiento en el Perú, por lo que se presenta el caso de un canino Cocker Spaniel, de tres meses de edad, con historia de regurgitación crónica de alimentos sólidos y bajo desarrollo. Al examen clínico se mostraba alerta y activo con condición corporal baja; el esófago a la palpación a nivel cervical se mostraba aparentemente flácido y notorio y a la compresión abdominal parecía distenderse. La imagen radiográfica contrastada reveló una notoria distención esofágica localizada delante de la imagen cardiaca, lo que dio el diagnóstico de un megaesófago pre-cardiaco. El diagnostico presuntivo fue la presencia del cuarto arco aórtico persistente, por lo que se sugirió a los propietarios la realización de la cirugía. Se realizó la cirugía exploratoria torácica confirmando el diagnóstico y se realizó la cirugía correctiva ligando y transectando el cuarto arco aórtico persistente. Se observó una rápida recuperación en el paciente, habiéndolo evaluado mensualmente durante un año sin presentar complicaciones.
\end{abstract}

Palabras clave: cuarto arco aórtico persistente; cirugía; perros; megaesófago

\section{AbSTract}

The persistent of the aortic right arch is a congenital anomaly that is considered difficult to treat in Peru, and therefore is presented the case of a three-month-old Cocker Spaniel canine with a history of chronic regurgitation of solid foods and poor

\footnotetext{
${ }^{1}$ Clínica de Animales Menores (CAM), Facultad de Medicina Veterinaria, Universidad Nacional Mayor de San Marcos, Lima, Perú

${ }^{2}$ Laboratorio de Bioquímica, Nutrición Animal y Alimentación Animal, Facultad de Medicina Veterinaria, Universidad Nacional Mayor de San Marcos, Lima, Perú

${ }^{3}$ E-mail: ichipayo@hotmail.com
}

Recibido: 12 de septiembre de 2018

Aceptado para publicación: 10 de abril de 2019 
development. At clinical examination he was alert and active but with low body condition; the esophagus on palpation at the cervical level was apparently flaccid and noticeable, and at abdominal compression it seemed to increase its volume. The contrasted radiographic image revealed a marked esophageal distension located in front of the cardiac image, which gave the diagnosis of a pre-cardiac megaesophagus. The presumptive diagnosis was the presence of the fourth persistent right aortic arch, so the surgery was suggested to the owners. Exploratory thoracic surgery was performed confirming the diagnosis and corrective surgery was performed by ligand and transecting the fourth persistent aortic arch. A rapid recovery was observed in the patient, and monthly evaluations for a year were done without detecting complications.

Key words: persistent aortic arch; surgery; dog; megaesophagus

\section{INTRODUCCIÓN}

El cuarto arco aórtico derecho persistente con presencia de un ligamento arterioso izquierdo (o ductus arterioso) es la forma más frecuente de anomalía del anillo vascular constrictor en perros (Helprey, 1979). En el desarrollo embrionario de los perros, el arco aórtico se desarrolla a partir del cuarto arco aórtico izquierdo y la aorta dorsal izquierda. Además, un vaso fetal, conocido como ductus arterioso, conecta la arteria pulmonar con la aorta, permitiendo que la sangre se desvíe de los pulmones inoperativos del feto. Después del nacimiento, el ductus arterioso se cierra y su remanente estructural se conoce como Ligamentum arteriosum (AL) (Nucci et al., 2018). Sin embargo, se pueden presentar alteraciones en el desarrollo del arco aórtico y la posición y permanencia de las estructuras vasculares asociadas a este pueden generar anillos de constricción capaces de alterar el correcto funcionamiento del esófago, disminuyendo su capacidad de distención y, de esa forma, limitando el pasaje del bolo alimenticio durante la deglución hacia el estómago.

En perros con cuarto arco aórtico persistente, es el cuarto arco aórtico derecho el que forma el arco aórtico en el lado derecho en lugar del izquierdo permitiendo que el esófago sea atrapado a nivel de la base del corazón con un anillo vascular compuesto por el cuarto arco aórtico persistente (derecha), corazón (ventral), arteria pulmonar (izquierda) y AL (izquierda y dorsal). A medida que el perro afectado crece, la compresión focal del esófago se desarrolla limitando su capacidad de ingesta de alimentos sólidos. Esto produce la regurgitación postprandial de los alimentos sólidos, siendo el principal signo de cuarto arco aórtico persistente en perros jóvenes (Buchanan, 2004)

Un diagnóstico presuntivo se puede hacer por medio de la radiografía de contraste, observándose el esófago dilatado cranealmente, en su región cervical o en el tercio craneal del tórax y estrecho en la base del corazón. El diagnóstico definitivo se realiza mejor por medio de la exploración quirúrgica (Muldoon et al., 1997).

El tratamiento definitivo para animales con cuarto arco persistente es la ligadura quirúrgica y la división de la parte del anillo vascular constrictivo (House et al., 2005). El componente constrictor del anillo vascular que se encuentra con mayor frecuencia es el ligamento arterioso izquierdo (Helphrey 1979; Ellison 1980; van Gundy 1989). Los casos raros requieren de la división de otras estructuras como arterias subclavias izquierdas aberrantes retroesofágicas, un conducto arterioso izquierdo o derecho y un arco aórtico izquierdo o derecho en casos de arcos aórticos dobles (Helphrey 1979; Ellison 1980; 


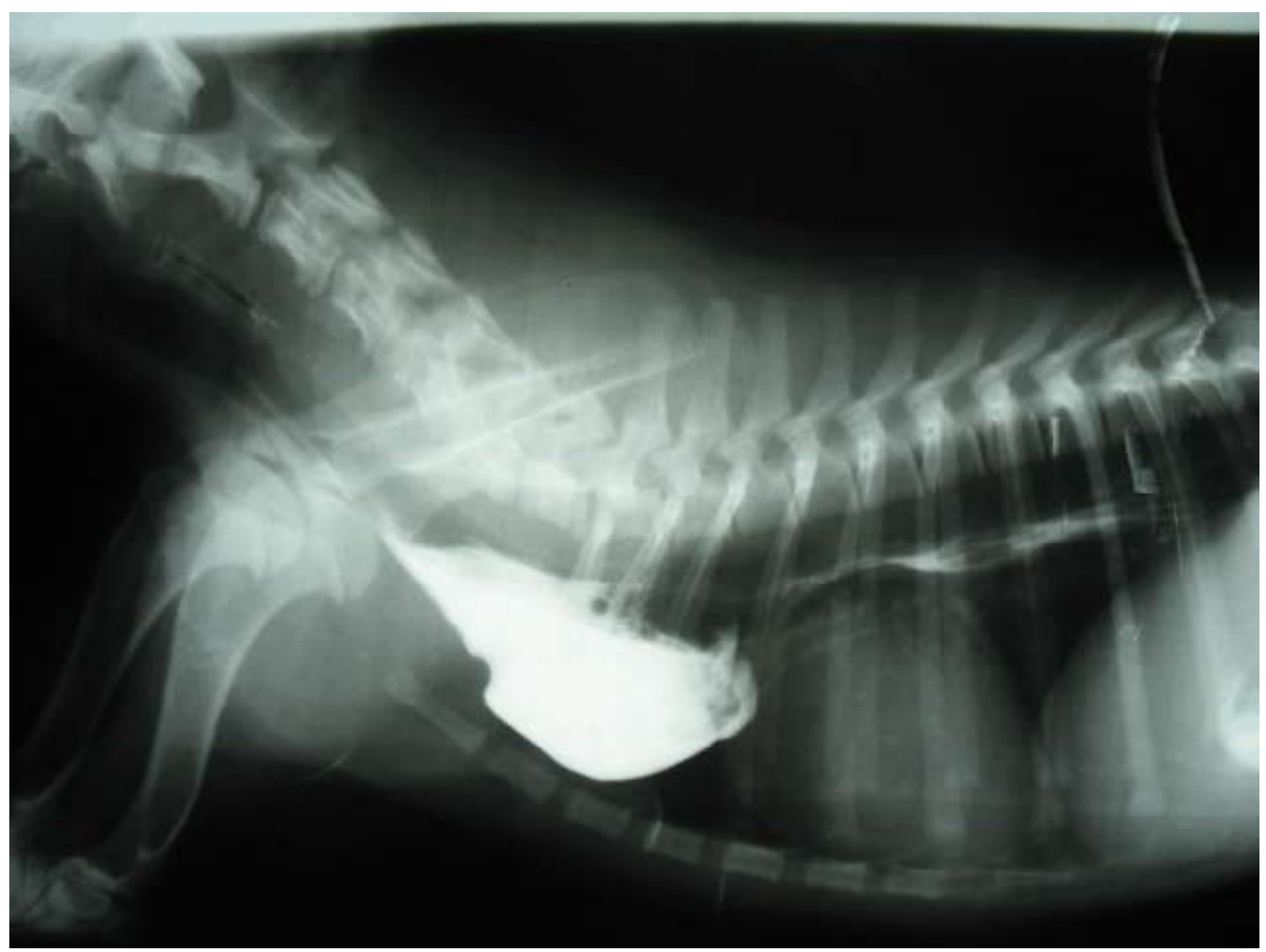

Figura 1. Tórax, vista laterolateral derecha, mostrando la presencia del megaesófago pre-cardiaco y el escaso pasaje de medio de contraste

van Gundy 1989; Holt et al. 2000). La evaluación preoperatoria, especialmente la angiografía, mejora la planificación del abordaje quirúrgico y la identificación de estructuras que requieren ligadura y división. El amplio conocimiento de la anatomía vascular facilita la identificación intraoperatoria precisa de la naturaleza del anillo vascular, particularmente en casos de configuraciones raras e inusuales (House et al., 2005).

En el presente artículo se informa del caso de un perro con cuarto arco aórtico persistente diagnosticado y tratado quirúrgicamente. El caso es interesante porque el animal estuvo acompañado durante un año sin alteraciones clínicas o secuelas detectables.

\section{Descripción del Caso}

\section{Evaluación del Paciente}

Al consultorio médico de la Clínica de Animales Menores de la Facultad de Medicina Veterinaria de la Universidad Nacional Mayor de San Marcos, Lima, se presentó un canino Cocker Spaniel, de tres meses de edad, hembra, con $4 \mathrm{~kg}$ de peso vivo, y con historia de regurgitación crónica de alimentos sólidos.

Los propietarios manifestaban que el paciente tenía un desarrollo menor al que presentaban sus hermanos de camada. Además, desde que empezó a comer material solido se observaba que el paciente devolvía los ali- 


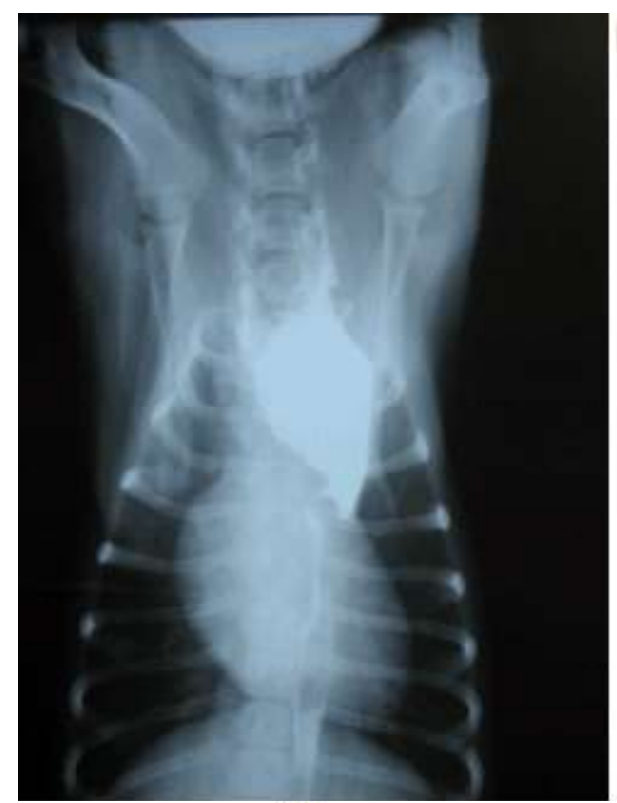

(a)

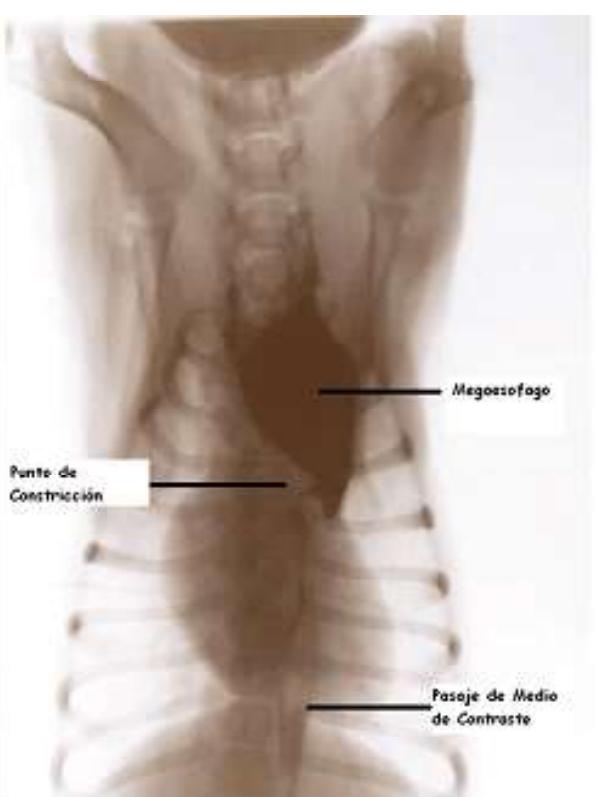

(b)

Figura 2. Tórax. a) Vista ventrodorsal mostrando el anillo de constricción esofágica en el hemitórax izquierdo y el escaso pasaje de medio de contraste; b) Negativización de la figura mostrando de manera clara el megaesófago y la estructura de constricción que genera la estenosis esofágica y la dilatación craneal

mentos ingeridos a los pocos minutos sin demasiado esfuerzo, por lo que su alimentación se basaba en dietas líquidas. El animal se presentaba con mucho apetito, pero la regurgitación limitaba su alimentación.

Las constantes fisiológicas se presentaron sin mayores anormalidades. El paciente en el examen clínico se mostró alerta y activo, aunque se observó una condición corporal baja (2/5). A la palpación, el esófago se mostraba aparentemente flácido y notorio y a la compresión abdominal parecía distenderse.

Se realizó el examen radiológico contrastado del esófago usando una suspensión líquida de sulfato de bario para uso oral (BarioDif ${ }^{\circledR} 98 \% \mathrm{p} / \mathrm{p}, 10 \mathrm{ml} / \mathrm{kg}$ de peso vivo). La imagen obtenida en proyección laterolateral derecha reveló una notoria distención esofágica localizada delante de la imagen cardiaca, lo que dio el diagnóstico de un megaesófago pre-cardiaco (Figura 1), lo que resulta compatible con la presencia de un anillo vascular constrictor del esófago.

La placa radiográfica en proyección ventrodorsal permitió la evaluación definitiva del megaesófago en su posición pre-cardiaca evidenciando también el pasaje de una pequeña cantidad de medio de contraste líquido hacia el estómago del paciente (Figura 2a). Además, se pudo notar el punto de constricción sobre el esófago en el lado izquierdo del tórax. La negativización de la imagen ventrodorsal (Figura $2 b$ ) permitió la notoria visualización de las estructuras antes mencionadas y facilitó la comprensión de la patología.

De acuerdo con la edad del paciente y las observaciones realizadas, el diagnóstico tentativo fue persistencia del cuarto arco aórtico derecho con formación de un anillo 


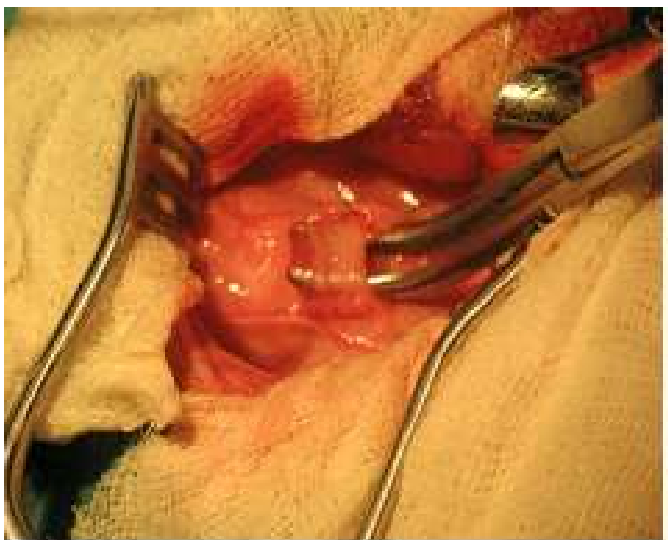

(a)

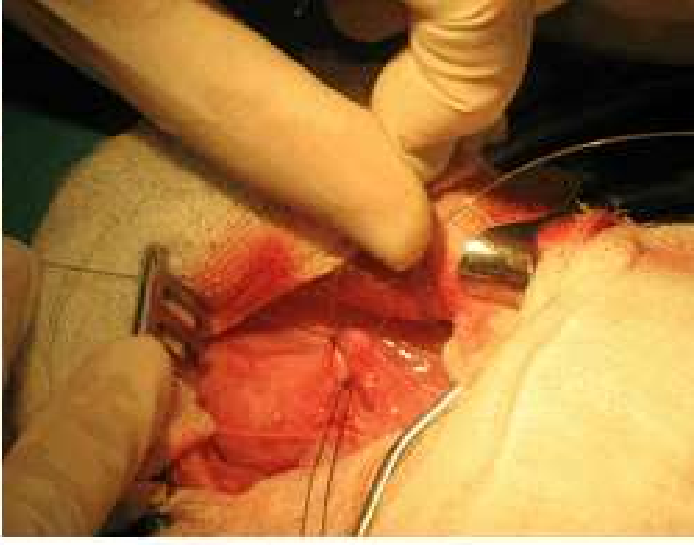

(b)

Figura 3. a) Abordaje torácico en $4^{\circ}$ espacio intercostal. Nótese la divulsión del anillo de constricción. b) Ligadura en proximal y distal del ligamento antes de su sección

vascular constrictor esofágico, por lo que se recomendó la cirugía exploratoria y correctiva.

\section{Técnica Quirúrgica Correctiva}

En el análisis pre-quirúrgico no se encontró anormalidades en los resultados de hematología, función hepática o renal. El protocolo anestésico consistió en la administración en forma combinada, vía i.m., de acepromazina $0.05 \mathrm{mg} / \mathrm{kg}$, fentanilo $0.005 \mathrm{mg} / \mathrm{kg}$ y atropina $0.045 \mathrm{mg} / \mathrm{kg}$, a fin de facilitar el manejo del paciente y como premedicación anestésica. Una vez obtenido el efecto de sedación se preoxigenó el paciente con una mascarilla y se colocó una vía endovenosa para fluido terapia de mantenimiento con suero fisiológico a una tasa de infusión de $10 \mathrm{ml} / \mathrm{kg}$ de p.v./hora. Se utilizó un protocolo de antibióticoterapia profiláctica con cefazolina $25 \mathrm{mg} / \mathrm{kg}$ vía endovenosa (Chipayo et al., 2018).

La inducción se realizó con midazolam $0.2 \mathrm{mg} / \mathrm{kg}$ y ketamina $5 \mathrm{mg} / \mathrm{kg}$, vía endovenosa y con ayuda del agente anestésico sevofluorano al 5\% con mascarilla. Una vez eliminado el reflejo deglutorio se procedió a la intubación y se conectó al equipo de anestesia inhalatoria en un circuito abierto de Bein.
El mantenimiento anestésico se realizó con sevofluorano (3-5\%) e infusión constante de fentanilo a $0.003 \mathrm{mg} / \mathrm{kg} / \mathrm{h}$. Se dio ventilación inspiratoria a presión positiva (VIPP) manual a fin de controlar la respiración en el paciente y facilitar la maniobra quirúrgica.

El paciente fue sometido a una toracotomía intercostal izquierda para eliminar el anillo de constricción y solucionar el impedimento mecánico de la deglución. Luego de elevar con delicadeza los planos musculares se procedió al ingreso a la cavidad torácica por el $4^{\circ}$ espacio intercostal para explorar la región pre-cardiaca, encontrar el anillo de constricción y seccionarlo. Se encontró como única anormalidad el anillo correspondiente al cuarto arco aórtico izquierdo a manera de una banda fibrosa no viable (sin aparente función vascular) (Figura 3a), el mismo que fue ligado con material de sutura absorbible de 2-0 (Figura 3b) y después seccionado. Se realizó adicionalmente la exploración de la superficie esofágica para descartar la presencia de bridas de tejido fibroso que continúen con el efecto de estenosis, pero estas no fueron determinadas. Posteriormente se evidenció la capacidad de pasaje a través del lumen esofágico mediante el uso de un tubo endotraqueal número 4 con el balón inflado. 


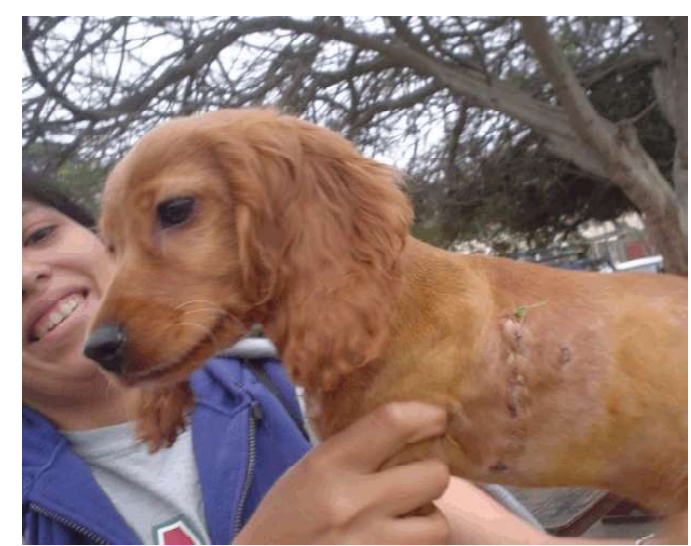

Figura 4. Paciente después de 10 días de la cirugía correctiva del cuarto arco aórtico persistente
Finalmente se procedió a la colocación de un tubo de toracotomía utilizando una sonda de Foley siliconada y se realizó el cierre de la pared torácica mediante el afrontamiento intercostal con suturas de nylon y la síntesis de los planos musculares en el orden inverso a lo realizado en el ingreso. Una vez terminada la sutura de los tejidos blandos, se acopló una llave de tres vías a la sonda de Foley para sellarla y devolverle la negatividad de la cavidad torácica. La sonda permaneció en su posición por dos días, protegida por un vendaje simple, y luego fue retirada por tracción simple. Terminado el abordaje se infiltró una solución de lidocaína $2 \%$ con epinefrina desde el $2^{\circ}$ al $6^{\circ}$ espacio intercostal a fin de mejorar la analgesia posoperatoria temprana. La analgesia posoperatoria se manejó con morfina, vía endovenosa, a las $2 \mathrm{~h}$ de terminada la cirugía y se mantuvo con tramadol cada $12 \mathrm{~h}$ por 4 días. La terapia antibiótica se realizó con cefalexina $25 \mathrm{mg} / \mathrm{kg}$, vía oral, cada $8 \mathrm{~h}$ por 7 días. La sutura de la piel fue retirada a los 10 días. En la Figura 4 se puede observar al paciente 10 días después de la cirugía, en estado normal.
La dieta posoperatoria consistió en líquidos por una semana, seguido de un alimento semisólido, siempre en posición vertical. Se realizaron evaluaciones mensuales del paciente durante un año, sin que se observasen complicaciones.

\section{Discusión}

Las anomalías del anillo vascular son malformaciones congénitas de los grandes vasos y estructuras asociadas (Ellison, 1980). Han sido reportadas en humanos (Lam et al., 1978), equinos (Bartels y Vaughan, 1969) y bovinos (Rooney y Watson, 1956), así como en caninos y felinos (Ellison, 1980), siendo el primer reporte de un caso canino de cuarto arco aórtico persistente a inicios del siglo pasado (Jex-Blake, 1926).

La anomalía que se encontró confirmó el diagnóstico presuntivo de persistencia del cuarto arco aórtico derecho con la formación de un anillo vascular. Los signos clínicos y la historia del paciente fueron consistentes con lo reportado en la literatura, y la examinación radiográfica y clínica fueron importantes para el diagnóstico.

El cuarto arco aórtico derecho persistente es una de las anomalías del anillo vascular más comúnmente diagnosticada en perros. Se han reportado siete patrones de anomalías del anillo vascular en el perro y el gato, y el arco aórtico derecho persistente representa el 95\% de los casos (Helphrey, 1979). En la práctica veterinaria en el Perú, encontrar casos de este tipo lleva en muchos casos a la eutanasia del paciente (Grandez et al., 2012); sin embargo, existe la posibilidad de realizar la cirugía correctiva y solucionar la anomalía, como se describe en el presente reporte. La edad al momento de la corrección quirúrgica es un factor importante en el pronóstico a largo plazo (Ellison, 1980). La intervención quirúrgica temprana es la principal recomendación, porque la dilatación 
esofágica y los trastornos de la motilidad pueden empeorar y posiblemente se volverían irreversibles si la cirugía se retrasa (Muldoon et al., 1997).

\section{Literatura Citada}

1. Bartels JE, Vaughan JT. 1969. Persistent right aortic arch in the horse. J Am Vet Med Assoc 154: 406-409.

2. Buchanan $\boldsymbol{J W}$. 2004. Tracheal signs and associated vascular anomalies in dogs with persistent right aortic arch. J Vet Intern Med 18: 510-514. doi: 10.1111/ j.1939-1676.2004.tb02576.x

3. Chipayo Y, Huerta C, Olazabal J, Diaz D. 2018. Tratamiento quirúrgico temprano de un caso de luxación patelar lateral bilateral en un paciente canino. Rev Inv Vet Perú 29: 720-724. doi: 10.15381/rivep.v29i2.14535

4. Ellison $G W$. 1980. Vascular ring anomalies in the dog and cat. Comp Cont Educ Pract 2: 693-705.

5. Grandez R, Bowler B, de Priego CM, Yi P, Torres L, Valencia R. 2012. Persistencia del arco aórtico derecho en Perro sin Pelo del Perú - reporte de un caso. Rev Inv Vet Perú 23: 523-528. doi: 10.15381/rivep.v23i4.974

6. Helphrey M. 1979. Vascular ring anomalies in the dog. Vet Clin N AmSmall 9: 207-218. doi: 10.1016/S01955616(79)50030-8

7. Holt D, Heldman E, Michel K, Buchanan JW. 2000. Esophageal obstruction caused by a left aortic arch and an aberrant right patent ductus arteriosus in two German Shepherd litter mates. Vet Surg 29: 264-270. doi: 10.1053/jvet.2000.4394

8. House AK, Summerfield NJ, German AJ, Noble PJM, Ibarrola P, Brockman DJ. 2005. Unusual vascular ring anomaly associated with a persistent right aortic arch in two dogs. J Small Anim Pract 46: 585-590. doi: 10.1111/j.17485827. 2005.tb00291.x

9. Jex-Blake. 1926. Obstruction of the oesophagus caused by a persistent ductus arteriosus. Lancet 28: 542-544. doi: 10.1016/S0140-6736(01)27830-1

10. Lam CR, Kabbani S, Arciniegas E. 1978. Symptomatic anomalies of the aortic arch. Surg Gynecol Obstet 147: 673-681.

11. Muldoon MM, Birchard SJ, Ellison $G W .1997$. Long-term results of surgical correction of persistent right aortic arch in dogs: 25 cases (1980-1995). J Am Vet Med Assoc 210: 1761-1763.

12. Nucci D, Hurst K, Monnet E. 2018. Retrospective comparison of short-term outcomes following thoracoscopy versus thoracotomy for surgical correction of persistent right aortic arch in dogs. J Am Vet Med Assoc 253: 444-451. doi: 10.2460/javma.253.4.444

13. Rooney JR, Watson DF. 1956. Persistent right aortic arch in a calf. J Am Vet Med Assoc 129: 5-7.

14. van Gundy T. 1989. Vascular ring anomalies. Comp Cont Educ Pract 11: $36-48$. 